\title{
ON A SUFFICIENT CONDITION FOR PROXIMITY
}

\author{
BY \\ KA-SING LAU ${ }^{1}$
}

\begin{abstract}
A closed subspace $M$ in a Banach space $X$ is called $U$-proximinal if it satisfies: $(1+\rho) S \cap(S+M) \subseteq S+\varepsilon(\rho)(S \cap M)$, for some positive valued function $\varepsilon(\rho), \rho>0$, and $\varepsilon(\rho) \rightarrow 0$ as $\rho \rightarrow 0$, where $S$ is the closed unit ball of $X$. One of the important properties of this class of subspaces is that the metric projections are continuous. We show that many interesting subspaces are $U$-proximinal, for example, the subspaces with the 2-ball property (semi $M$-ideals) and certain subspaces of compact operators in the spaces of bounded linear operators.
\end{abstract}

1. Introduction. We call a closed subspace $M$ of a real Banach space $X$ an $M$-ideal if the annihilator $M^{\perp}$ of $M$ is an $L$-summand in $X^{*}$. This notion was formulated and studied by Alfsen and Effros [1]. It was proved that if $M$ is an $M$-ideal, then $M$ is a proximinal subspace of $X$ [1], [5]. In [4], Hennefeld showed that the space of compact operators on $l^{p}\left(\right.$ or $\left.c_{0}\right), 1<p<\infty$, is an $M$-ideal in the space of bounded linear operators on $l^{p}$ (or $c_{0}$ respectively). This theorem is also true for operators from $l^{p}$ into $l^{q}, 1<p<q<\infty$ [11]. (It is well known that if $1 \leqslant q<p<\infty$, then every bounded linear operator from $l^{p}$ into $l^{q}$ is compact.) $M$-ideal theory provides a convenient tool to study the approximation of operators by the space of compact operators and has been investigated by many authors [3], [4], [5], [9], [11], [15], [16]. However, in some cases, the class of $\boldsymbol{M}$-ideals appears to be too restricted; for example, the space of compact operators on $l^{1}$ is not an $M$-ideal in the space of bounded linear operators on $l^{1}$ [16]. It is our attempt to consider another sufficient condition for proximity which preserves certain important properties of $\boldsymbol{M}$-ideals and also includes some other interesting classes of proximinal subspaces.

Motivated by a lemma of Holmes in [5], we call a closed subspace $M$ of a Banach space $X U$-proximinal if there exists a positive function $\varepsilon(\rho), \rho>0$, with $\varepsilon(\rho) \rightarrow 0$ as $\rho \rightarrow 0$ and satisfies

$$
(1+\rho) S \cap(S+M) \subseteq S+\varepsilon(\rho)(S \cap M), \quad \rho>0,
$$

Received by the editors February 3, 1978 and, in revised form, July 24, 1978.

AMS (MOS) subject classifications (1970). Primary 41A65; Secondary 46B99.

Key words and phrases. Compact operators, measurable functions, $M$-ideals, proximity, uniformly convex.

${ }^{1}$ Supported in part by the Faculty of Arts and Sciences research grant of the University of Pittsburgh. 
where $S$ denotes the closed unit ball of $X$. Examples of $U$-proximinal subspaces are:

(i) $X$ is uniformly convex and $M$ is a closed subspace of $X$;

(ii) $X=B(K)$, the space of bounded functions on a topological space $K$ and $M=C(K)$, the space of bounded continuous functions on $K$;

(iii) $M$ is a semi $M$-ideal or semi $L$-summand in a Banach space $X$;

(iv) $X=L^{\infty}\left(\Omega, L^{1}\right)$, the space of bounded Bochner measurable functions from a $\sigma$-finite measure space $\Omega$ into $L^{1}$ and $M$ is the subspace of $f$ in $X$ such that $f(\Omega)$ is weakly precompact. In particular, if we let $L(E, F)(K(E, F))$ denote the space of bounded linear operators (compact operators, respectively), then $K\left(L^{1}(\Omega), l^{1}\right)$ is proximinal in $L\left(L^{1}(\Omega), l^{1}\right)$.

Our paper is divided into six sections. In $\$ 2$, we define some basic terminologies and give several reformulations of the definition of $U$ proximity. The metric projection from $X$ into a proximinal subspace $M$ is the map $P$ which sends $x \in X$ to the set of best approximations from $M$ to $x$. The study of the continuity of metric projections is an important component of the theory of best approximation. In $\$ 3$, we show that if $M$ is $U$ proximinal, then the metric projection $P$ is continuous (with respect to the Hausdorff metric in the range). We also give a condition for $\boldsymbol{P}$ to be Lipschitz continuous. In $\$ \$ 4-6$, we show that the examples listed above are in fact $U$-proximinal subspaces.

Acknowledgement. The author is indebted to Professor T. A. Metzger for some suggestions in the first draft. He would also like to thank the referee for bringing his attention to several recent publications in this subject.

2. Definitions and preliminaries. Let $X$ be a real Banach space, let $S_{r}(X)$ (or $S_{r}$ ) denote the closed ball of radius $r$ centered at $0 ; S_{1}(X)=S(X)\left(S_{1}=S\right)$. If $M$ is a closed subspace of $X$, then for $x \in X$, we denote the subspace generated by $M$ and $x$ by $\langle M, x\rangle$, and denote $S_{r}(\langle M, x\rangle)$ by $S_{r}[x]$.

Let $F(X)$ be a family of nonempty bounded closed subsets of $X$. For any $A, B \in F(X)$, we define

$$
d_{H}(A, B)=\inf \left\{r: A \subseteq B+S_{r} \text { and } B \subseteq A+S_{r}\right\}
$$

Then $d_{H}$ is a metric on $F(X)$ and is called the Hausdorff metric.

For $x \in X$ and for any subset $A$ in $X$, we define another distance function $d(x, A)=\inf \{\|x-z\|: z \in A\}$. A point $y$ in a closed subspace $M$ of $X$ is called a best approximation from $M$ to $x$ if $\|x-y\|=d(x, M) . M$ is called a proximinal subspace of $X$ if every $x \in X$ has a best approximation from $M$. Propositions 2.2 and 2.3 will give the motivation of a simple sufficient condition for $M$ to be a proximinal subspace. 
LEMMA 2.1. Let $g$ be a concave function defined on $[0,1]$ with $g(0)>0$ and $g(1)=0$. Let $h$ be a function defined on $[0, a], a>1$, with $h(x)=a g(x / a)$, $x \in[0, a]$. Then $h(1)>h(x)-g(x)$ for $0<x<1$.

Proof. Note that the derivatives $h^{\prime}(x), g^{\prime}(x)$ exist and decrease almost everywhere. Hence

$$
h^{\prime}(x)-g^{\prime}(x)=g^{\prime}\left(\frac{x}{a}\right)-g^{\prime}(x)>0 \text { a.e. }
$$

and

$$
h(1)=h(1)-g(1) \geqslant h(x)-g(x), \quad 0<x<1 .
$$

Let $X$ be a two dimensional normed linear space. For $\rho>0$, let $L$ be a line which is tangent to $S$ at $w$ and cuts the sphere $\{z:\|z\|=1+\rho\}$ at $x$ and $y$. Let $\left(x^{\prime}: y^{\prime}\right)$ be any open line segment which is parallel to $L$, with $\left\|x^{\prime}\right\|=1+$ $\rho,\left\|y^{\prime}\right\|=1$ and does not intersect $S$. Lemma 2.1 implies that $\left\|x^{\prime}-y^{\prime}\right\|<$ $\max \{\|x-w\|,\|y-w\|\}$.

Let $M$ be a proximinal subspace in $X$, let $x \in X \backslash M$ and let $X_{1}=\langle M, x\rangle$. Let $f \in X_{1}^{*}$ be such that $f^{-1}(0)=M,\|f\|=1$ and define

$$
\alpha(x, \rho)=d_{H}\left((1+\rho) S[x] \cap f^{-1}(1), S[x] \cap f^{-1}(1)\right), \quad \rho>0 .
$$

It follows from simple geometry that for each $x \in X \backslash M$,

$$
\left|\alpha(x, \rho)-\alpha\left(x, \rho^{\prime}\right)\right|<\frac{2\left|1+\rho+\rho^{\prime}\right|}{\rho}\left|\rho-\rho^{\prime}\right| \text { for } \rho>0 .
$$

Hence $\alpha(x, \cdot)$ is continuous on $\mathbf{R}^{+}$.

Proposition 2.2. Let $M$ be a proximinal subspace of a Banach space $X$ and let $\alpha(x, \rho)$ be defined as above. Then

$$
\alpha(x, \rho)=\inf \{r>0:(1+\rho) S \cap(S[x]+M) \subseteq S+r S(M)\} .
$$

Proof. Without loss of generality, we may assume that $X=\langle M, x\rangle$. It is clear from the definition of $\alpha(x, \rho)$ that

$$
\alpha(x, \rho)<\inf \{r>0:(1+\rho) S \cap(S+M) \subseteq S+r S(M)\} .
$$

To prove the reverse inequality, let $0<\varepsilon<1$ and let $y \in(1+\rho) S \cap(S+$ $M)$. We will consider the case $y \notin M$ first. Assume $f(y)>0$ (the case $f(y)<0$ is similar), and let $y_{1}=t y \in f^{-1}(1)$ for some $t>0$. There exists $w_{1} \in S \cap f^{-1}(1)$ such that

$$
\left\|y_{1}-w_{1}\right\| \leqslant d\left(y_{1}, S \cap f^{-1}(1)\right)+\varepsilon .
$$

If $\left\|y_{1}\right\|<1+\rho$, we define $x_{1}=y_{1}$, otherwise we define $x_{1}$ to be the point on the line segment of $\left(y_{1}: w_{1}\right)$ with $\left\|x_{1}\right\|=1+\rho$. In either case, it can be shown that $\left\|x_{1}-w_{1}\right\|<\alpha(x, \rho)+\varepsilon$. By the remark before the proposition, 
we can choose a $z \in f^{-1}(f(y)) \cap\{w:\|w\|=1\}$ such that $\|y-z\|<$ $\alpha(x, \rho)+\varepsilon$. Hence

$$
y=z+(y-z) \in S+(\alpha(x, \rho)+\varepsilon) S(M) .
$$

If $y \in M$, we can choose $y^{\prime} \in(1+\rho) S \backslash M$ with $\left\|y^{\prime}-y\right\|<\varepsilon / 2(1+\rho)$ and $z^{\prime} \in f^{-1}\left(f\left(y^{\prime}\right)\right) \cap S$ satisfies (1). Let

$$
z^{\prime \prime}=\left\|z^{\prime}-\left(y^{\prime}-y\right)\right\|^{-1}\left(z^{\prime}-\left(y^{\prime}-y\right)\right) .
$$

Then $z^{\prime \prime}$ is in $M$ since

$$
f\left(z^{\prime}-\left(y^{\prime}-y\right)\right)=f\left(z^{\prime}\right)-f\left(y^{\prime}\right)=0 .
$$

That

$$
\left\|y-z^{\prime \prime}\right\|<\varepsilon+(1+\varepsilon)(\alpha(x, \rho)+\varepsilon)=\beta
$$

implies that

$$
y=z^{\prime \prime}+\left(y-z^{\prime \prime}\right) \in S+\beta S(M) .
$$

Since this is also true for the $y \notin M$ as in (1) and since $\varepsilon$ is arbitrary, we conclude that

$$
\alpha(x, \rho)>\inf \{r>0:(1+\rho) S \cap(S+M) \subseteq S+r S(M)\} .
$$

The following proposition is the foundation of this paper; the proof is similar to [5, Lemma 2].

Proposition 2.3. Let $M$ be a closed subspace of $X$ and let $x \in X \backslash M$. Suppose there exists a function $\varepsilon: \mathbf{R}^{+} \rightarrow \mathbf{R}^{+}$(depending on $x$ ) such that $\varepsilon(\rho) \rightarrow 0$ as $\rho \rightarrow 0$ and

$$
(1+\rho) S \cap \overline{(S[x]+M)} \subseteq \overline{S+\varepsilon(\rho) S(M)}, \quad \rho>0 .
$$

Then $x$ has a best approximation from $M$.

Furthermore, if $\|x\|<1+\rho_{0}$ and if $\inf \{\|x-z\|: z \in M\}<1$. Then given any $r>\varepsilon\left(\rho_{0}\right)$, there exists a best approximation $z_{0}$ in $M$ such that $\left\|z_{0}\right\|<r$.

Proof. Without loss of generality, assume that $\|x\|=1+\rho_{0}$ and inf $\{\| x-$ $z \|: z \in M\}=1$. We claim that $x \in \overline{S[x]+M}$. Indeed, a sequence $\left\{z_{n}\right\}$ in $M$ can be chosen such that $\left\|x-z_{n}\right\| \rightarrow 1$. Let

$$
x_{n}=\left\|x-z_{n}\right\|^{-1}\left(x-z_{n}\right)+z_{n},
$$

then the sequence $\left\{x_{n}\right\}$ is contained in $S[x]+M$ and $x_{n} \rightarrow x$.

Choose a sequence of positive numbers $\left\{\rho_{n}\right\}$ such that $\rho_{n} \rightarrow 0$ and $\sum_{n=0}^{\infty} \varepsilon\left(\rho_{n}\right)<r$ where $r>\varepsilon\left(\rho_{0}\right)$. By hypothesis there exists $z_{1} \in \varepsilon\left(\rho_{0}\right) S(M)$ with $\left\|x-z_{1}\right\| \leqslant 1+\rho_{1}$. Note that $x-z_{1}$ is also in $\overline{S[x]+M}$, the same argument yields a $z_{2}$ in $\varepsilon\left(\rho_{1}\right) S(M)$ with $\left\|x-z_{1}-z_{2}\right\|<1+\rho_{2}$. Inductively, we can find a sequence $\left\{z_{n}\right\}$ such that $z_{n} \in \varepsilon\left(\rho_{n-1}\right) S(M)$ and $\left\|x-\sum_{k=1}^{n} z_{k}\right\|$ $<1+\rho_{n}$. Let $z_{0}=\sum_{n=1}^{\infty} z_{n}$, then $\left\|z_{0}\right\|<\sum_{n=0}^{\infty} \varepsilon\left(\rho_{n}\right)<r$ and $\left\|x-z_{0}\right\|=1$. This completes the proof. 
Proposition 2.4. Let $M$ be a closed subspace in $X$ and let $x \in X \backslash M$. Then the following conditions are equivalent:

(i) there exists an $\varepsilon: \mathbf{R}^{+} \rightarrow \mathbf{R}^{+}$such that $\varepsilon(\rho) \rightarrow 0$ as $\rho \rightarrow 0$ and

$$
(1+\rho) S \cap \overline{(S[x]+M)} \subseteq \overline{S+\varepsilon(\rho) S(M)}, \quad \rho>0 .
$$

(ii) there exists an $\varepsilon^{\prime}: \mathbf{R}^{+} \rightarrow \mathbf{R}^{+}$such that $\varepsilon^{\prime}(\rho) \rightarrow 0$ as $\rho \rightarrow 0$ and

$$
(1+\rho) S \cap(S[x]+M) \subseteq S+\varepsilon^{\prime}(\rho) S(M), \quad \rho>0 .
$$

(iii) there exists an $\varepsilon: \mathbf{R}^{+} \rightarrow \mathbf{R}^{+}$such that $\tilde{\varepsilon}$ is increasing, continuous, $\tilde{\varepsilon}(\rho) \rightarrow 0$ as $\rho \rightarrow 0$ and

$$
(1+\rho) S \cap(S[x]+M) \subseteq S+\tilde{\varepsilon}(\rho) S(M), \quad \rho>0 .
$$

Moreover, if one of the three conditions holds, the function $\alpha(x, \rho)$ defined in Proposition 2.2 converges to zero as $\rho \rightarrow 0$.

Proof. (i) $\Rightarrow$ (ii). Let $y \in(1+\rho) S \cap(S[x]+M)$. By Proposition 2.3 if $r=2 \varepsilon(\rho)$, then there exists a $z \in M$ such that $\|y-z\|<1$ and $\|z\|<2 \varepsilon(\rho)$. Let $\varepsilon^{\prime}(\rho)=2 \varepsilon(\rho)$, then $y \in S+\varepsilon^{\prime}(\rho) S(M)$ and (ii) follows. (ii) $\Rightarrow$ (i) is clear. To prove (ii) $\Rightarrow$ (iii), we can take $\tilde{\varepsilon}(\rho)=\alpha(x, \rho)+\rho$, then $\tilde{\varepsilon}$ is an increasing, continuous function. Proposition 2.2 implies that $\alpha(x, \rho)<\tilde{\varepsilon}(\rho)$. Hence $\tilde{\varepsilon}(\rho)$ $\rightarrow 0$ as $\rho \rightarrow 0$. That (iii) $\Rightarrow$ (ii) is obvious.

Definition 2.5. Let $M$ be a closed subspace of a Banach space $X$, we say that $M$ is locally $U$-proximinal if there exists a function $\varepsilon:(X \backslash M) \times \mathbf{R}^{+} \rightarrow \mathbf{R}^{+}$ such that for each fixed $x, \varepsilon(x, \cdot)$ is continuous, increasing on $\rho, \varepsilon(x, \rho) \rightarrow 0$ as $\rho \rightarrow 0$ and

$$
(1+\rho) S \cap(S[x]+M) \subseteq S+\varepsilon(x, \rho) S(M), \quad x \in M, \quad \rho>0 .
$$

$M$ is called $U$-proximinal if the function $\varepsilon$ can be chosen independent of $x$ and

$$
(1+\rho) S \cap(S+M) \subseteq S+\varepsilon(\rho) S(M) .
$$

It follows from Proposition 2.3 that locally $U$-proximinal and $U$-proximinal subspaces are proximinal.

3. Metric projections. Let $M$ be a proximinal subspace of $X$, for each $x \in X$, we define $P(x)$ to be the set of best approximations from $M$ to $x$. We call the map $P$ from $X$ into $F(M)$, the family of bounded closed subsets in $M$, as the metric projection from $X$ into $M$.

LEMMA 3.1. Let $M$ be a locally $U$-proximinal subspace in $X$. For each $x \in X \backslash M$, let $r_{x}=d(x, M)$. Then

$$
M \cap\left(S_{(1+\rho) r_{x}}+x\right) \subseteq P(x)+r_{x} \varepsilon(x, \rho) S(M), \quad \rho>0 .
$$

Proof. Without loss of generality, we assume that $r_{x}=1$. For $y \in M \cap$ $\left(S_{(1+\rho)}+x\right)$, it follows that $\|x-y\|<1+\rho$. That

$$
x-y=(x-z)+(z-y)
$$


where $z$ is the best approximation of $x$, implies that $x-y \in S+M$. Hence

$$
(x-y) \in(1+\rho) S \cap(S+M) .
$$

Since $M$ is locally $U$-proximinal, $x-y=w+v$ for some $w$ in $S$ and $v$ in $\varepsilon(x, \rho) S(M)$. Note that $(x-w)$ is in $M$ and, in fact, a best approximation to $x$ (for $1<\|x-(x-w)\|=\|w\|<1)$. Hence

$$
y=(x-w)-v \in P(x)+\varepsilon(x, \rho) S(M) .
$$

LEMMA 3.2. Let $M$ be a locally $U$-proximinal subspace in $X$. Then for any $x$, $y \in X \backslash M$ with $\|x-y\|<\rho$,

$$
d_{H}(P(x), P(y))<\max \left\{r_{x} \varepsilon\left(x, \frac{2 \rho}{r_{x}}\right), r_{y} \varepsilon\left(y, \frac{2 \rho}{r_{y}}\right)\right\} .
$$

PROOF. Since $r_{y}<r_{x}+\rho$ and

$$
\left(S_{r_{y}}+y\right) \subseteq\left(S_{r_{x}+2 \rho}+x\right),
$$

it follows that

$$
P(y) \subseteq M \cap\left(S_{r_{x}+2 \rho}+x\right) .
$$

By Lemma 3.1, we have

$$
P(y) \subseteq P(x)+r_{x} \varepsilon\left(x, \frac{2 \rho}{r_{x}}\right) S(M) .
$$

A similar argument yields that

$$
P(x) \subseteq P(y)+r_{y} \varepsilon\left(y, \frac{2 \rho}{r_{y}}\right) S(M),
$$

and the lemma follows easily from these inclusions and the definition of the Hausdorff metric on $F(M)$.

THEOREM 3.3. Let $M$ be a locally $U$-proximinal subspace in $X$. Suppose for each $\rho>0, \varepsilon(\cdot, \rho)$ is an upper semicontinuous function on $X \backslash M$. Then $P$ is a continuous function from $X$ into $F(M)$.

Proof. It is easy to show that $P$ is continuous for $x \in M$. Let $x \in X \backslash M$, for any $\delta>0$, there exists an $\eta$ in $(0,1)$ such that $\varepsilon(x, \eta)<\delta / 2$. Since $\varepsilon(\cdot, \eta)$ is upper semicontinuous, there exists a $\rho, 0<\rho<\left(\eta \cdot r_{x}\right) / 4$, such that for $\|x-y\|<\rho, \varepsilon(y, \eta)<\varepsilon(x, \eta)+\delta / 2$. Hence for $\|x-y\|<\rho$, we have

$$
\begin{aligned}
d_{H}(P(x), P(y)) & <\max \left\{r_{x} \varepsilon\left(x, \frac{2 \rho}{r_{x}}\right), r_{y} \varepsilon\left(y, \frac{2 \rho}{r_{y}}\right),\right\} \\
& <2 r_{x} \max \left\{\varepsilon\left(x, \frac{2 \rho}{r_{x}}\right), \varepsilon\left(y, \frac{4 \rho}{r_{x}}\right)\right\} \\
& <2 r_{x} \delta .
\end{aligned}
$$

This shows that $P$ is continuous at $x$. 
THEOREM 3.4. Let $M$ be a $U$-proximinal subspace of $X$. Then the metric projection $P: X \rightarrow F(M)$ is continuous.

Proof. In this case, we have $\varepsilon(x, \rho)=\varepsilon(y, \rho)=\varepsilon(\rho)$ for any $x, y \in X \backslash M$. Theorem 3.3 implies that $P$ is continuous.

COROllary 3.5. Let $M$ be a $U$-proximinal subspace of $X$. Then the metric projection $P$ admits a continuous selection s: $X \rightarrow M($ i.e., $s(x) \in P(x))$.

The following also follows easily from Lemma 3.2:

THEOREM 3.6. Let $M$ be a subspace of $X$. Suppose there exists $a k>0$ such that

$$
(1+\rho) S \cap(S+M) \subseteq S+k \rho S(M), \quad \rho>0 .
$$

Then the metric projection $P: X \rightarrow F(M)$ is a Lipschitz continuous function with Lipschitz constant not greater than $2 k$.

In the next three sections, we will see that many interesting examples will satisfy the above inclusion, We remark that the converse of the theorem is not true; for example, let $M$ be a closed subspace of a Hilbert space $X$, then the metric projection $P: X \rightarrow M$ is a Lipschitz function but $\varepsilon(\rho) \geq \sqrt{\rho^{2}+2 \rho}$. We also remark that a $U$-proximinal subspace may not have a uniformly continuous metric projection. Examples (certain closed subspaces in some uniformly convex spaces) can be found in [7], [14]. In general, a set valued function which satisfies the Lipschitz condition does not admit Lipschitz selection; it will be interesting to investigate this question for the metric projections in Theorem 3.6.

4. Some examples. In this section, we will give some simple examples of locally $U$-proximinal and $U$-proximinal subspaces.

Proposition 4.1. Let $M$ be a finite dimensional subspace in a Banach space $X$. Then $M$ is locally $U$-proximinal.

Proof. Let $x \in X \backslash M$ and assume that $X=\langle M, x\rangle$. Let $\varepsilon(x, \rho)=\alpha(x, \rho)$ $+\rho$, then it follows from the compactness of the unit ball that $\varepsilon(x, \rho) \rightarrow 0$ as $\rho \rightarrow 0$ and by Proposition 2.2,

$$
(1+\rho) S \cap(S+M) \subseteq S+\varepsilon(x, \rho) S(M) .
$$

A Banach space $X$ is called locally uniformly convex if for each $x \in X$ with $\|x\|=1$ and for any $\eta>0$, there exists a $\delta>0$ such that for any $y \in X$ with $\|y\| \leqslant 1$ and $\|x-y\| \geqslant \eta,\|x+y\|<2(1-\delta)$. $X$ is called uniformly convex if the $\delta$ can be chosen independent of $x$.

Proposition 4.2. Let $X$ be a locally uniformly convex space. Then every proximinal subspace is locally $U$-proximinal. 
Proof. Let $M$ be a proximinal subspace of $X$ and let $x \in X \backslash M$. Let $X_{1}=\langle M, x\rangle$ and let $f \in X_{1}^{*}$ with $f^{-1}(0)=M$ and $\|f\|=1$. If $y \in S$ with $f(y)=1$ and $\alpha(x, \rho)$ is defined as in Proposition 2.2, then

$$
\begin{aligned}
\alpha(x, \rho) & =d_{H}\left((1+\rho) S[x] \cap f^{-1}(1),\{y\}\right) \\
& <\operatorname{diam}\left((1+\rho) S[x] \cap f^{-1}(1)\right) .
\end{aligned}
$$

By the locally uniformly convexity of the norm on $X_{1}$, we can show that the last term approaches to zero as $\rho \rightarrow 0$. Let $\varepsilon(x, \rho)=\alpha(x, \rho)+\rho$; it follows that $M$ is locally $U$-proximinal.

Proposition 4.3. Let $X$ be a uniformly convex space. Then every closed subspace is $U$-proximinal.

Proof. Let $|L|$ denote the length of a line segment $L$ and define

$$
\varepsilon(\rho)=\sup \{|L|: L \text { is in }(1+\rho) S \backslash S\}+\rho \text {. }
$$

It is clear that $\varepsilon(\rho) \rightarrow 0$ as $\rho \rightarrow 0$ and $\varepsilon(\rho)>\alpha(x, \rho)+\rho$ where $\alpha(x, \rho)$ is defined as in Proposition 2.2. Hence

$$
(1+\rho) S \cap(S+M) \subseteq S+\varepsilon(\rho) S(M)
$$

and the proof is completed.

Let $K$ be a topological space, let $B(K)$ be the space of bounded continuous functions on $K$ with the supremum norm and let $C(K)$ be the subspace of bounded continuous functions in $B(K)$. It is well known that $C(K)$ is a proximinal subspace in $B(K)$ [6].

Proposition 4.4. Let $M$ be a closed subspace in $X=B(K)$ such that for each $h \in M, h \wedge \rho, h \vee(-\rho), \rho>0$, are also in $M$. Then $M$ satisfies

$$
(1+\rho) S \cap(S+M) \subseteq S+\rho S(M), \quad \rho>0 .
$$

Thus $M$ is $U$-proximinal, and in particular, $C(K)$ is $U$-proximinal.

Proof. For any $f \in(1+\rho) S \cap(S+M)$, we can write $f=g+h$ where $\|g\| \leqslant 1$ and $h \in M$. Let $h^{\prime}=(h \wedge \rho) \vee(-\rho)$; then $h^{\prime} \in M$ and $\left\|h^{\prime}\right\| \leqslant \rho$. Let $g^{\prime}=f-h^{\prime}$, we need only show that $\left\|g^{\prime}\right\|<1$ and hence

$$
f=g^{\prime}+h^{\prime} \in S+\rho S(M) \text {. }
$$

If $|h(x)| \leqslant \rho$, then $\left|g^{\prime}(x)\right|=|g(x)| \leqslant 1$. If $h(x)>\rho$, then three cases arise:

(i) if $g(x) \geqslant 0$, then $\left|g^{\prime}(x)\right|=f(x)-\rho \leqslant 1$;

(ii) if $g(x)<0$ and $f(x) \geqslant 0$, then

$$
\left|g^{\prime}(x)\right|=|f(x)-\rho| \leqslant(1+\rho)-\rho=1 ;
$$

(iii) if $g(x)<0$ and $f(x)<0$, then $|g(x)|=|f(x)|+h(x)$. This implies $|f(x)|<1-\rho$ and 


$$
\left|g^{\prime}(x)\right|=|f(x)-\rho| \leqslant|f(x)|+\rho<1 .
$$

If $h(x)<-\rho$, a similar proof shows that $\left|g^{\prime}(x)\right|<1$. Hence $\left\|g^{\prime}\right\|<1$ and the proof is completed.

5. Semi- $M$-ideals. A convex subset $F$ in a convex set $K$ is a face of $K$ if $\lambda y+(1-\lambda) z \in F, y, z \in K, 0<\lambda<1$ implies $y, z \in F$. Let $X$ be a Banach space and let $x \in S$ with $\|x\|=1$, we use face $(x)$ to denote the maximal proper face in $S$ containing $x$.

Let $J$ be a closed subspace of a Banach space $X$ and let

$$
J^{\prime}=\left\{x \in X: x=0 \text { or face }\left(\frac{x}{\|x\|}\right) \cap J=\varnothing\right\} .
$$

$J$ is called a semi-L-summand if each $x \in X$ has a unique decomposition as $x=y+z$ with $y \in J, z \in J^{\prime}$ and $\|x\|=\|y\|+\|z\| . J$ is called an $L$ summand if $J^{\prime}$ is a closed linear subspace of $X$. For detailed discussion of these and the semi- $M$-ideals, $M$-ideals, which we will define later, we refer the readers to [1], [12]. It follows directly from the definition that

$$
S=\operatorname{conv}\left((S \cap J) \cup\left(S \cap J^{\prime}\right)\right) \text {. }
$$

THEOREM 5.1. Every semi-L-summand $J$ in a Banach space $X$ is $U$-proximinal.

Proof. Let $x \in(1+\rho) S \cap(S+J)$ and $\|x\|=1+\rho$; then $x=(1+\rho)(y$ $+z$ ) with $y \in S \cap J$ and $z \in S \cap J^{\prime}$. Note that $x \in S+J, d(x, J)<1$. This implies $\|(1+\rho) z\| \leqslant 1$. Also note that $(1+\rho)-\rho /\|y\|>0$ (for otherwise, $(1+\rho)\|y\|<\rho$ will imply $(1+\rho)\|y\|+(1+\rho)\|z\|<1+\rho)$. Let $y^{\prime}=((1+\rho)-\rho /\|y\|) y$ and write $x=\left(y^{\prime}+(1+\rho) z\right)+\rho y /\|y\|$. Then $\| y^{\prime}$ $+(1+\rho) z \|=1$ and hence $x \in S+\rho(S \cap J)$.

Let $M$ be a closed subspace of a Banach space $X$. $M$ is called a semi-Mideal $(M$-ideal $)$ if $M^{\perp}=\left\{x^{*} \in S^{*}: x^{*}(x)=0 \forall x \in M\right\}$ is a semi-Lsummand ( $L$-summand, respectively) in $X^{*} . M$ is said to have the $n$-ball property if for any $\varepsilon>0$ and for any $n$ intersecting balls $S_{i}\left(a_{i}, r_{i}\right)=\{x$ : $\left.\left\|x-a_{i}\right\| \leqslant r_{i}\right\}$ with $S_{i}\left(a_{i}, r_{i}\right) \cap M \neq \varnothing$, then $\cap_{i=1}^{n} S_{i}\left(a_{i}, r_{i}+\varepsilon\right) \cap M \neq \varnothing$. It was proved that $M$ is a semi- $M$-ideal ( $M$-ideal) if and only if $M$ has the 2-ball ( $n$-ball, $n>3$, respectively) property [1], [12]. In [5], Holmes showed that $M$-ideals are $U$-proximinal. In the following, we will show that his theorem also holds for semi- $M$-ideals.

LemMa 5.2. Let $X$ be a Banach space and let $e^{*} \in X^{*}$ with $\left\|e^{*}\right\|=1$. Let $F=\left\{x \in S: e^{*}(x)=1\right\}$. Suppose $S=\operatorname{conv}(F \cup-F)$. Then $M=\left\{x: e^{*}(x)\right.$ $=0\}$ satisfies

$$
(1+\rho) S \cap(S+M) \subseteq S+\rho S(M) .
$$


Proof. Let $x \in(1+\rho) S \cap(S+M)$ with $\|x\|=1+\rho$. By assumption, there exist $a, b \in(1+\rho) F$ such that $x \in[a:-b]$. Let $y=(\rho /(1+\rho))((a-$ b)/2). It is easy to show that $x=(x-y)+y \in S+\rho S(M)$.

LEMMA 5.3. Let $J$ be a one dimensional semi-L-summand in $X$. Let $e \in J$ with $\|e\|=1$. Then $e$ is an extreme point of $S$. Moreover, for any $x \in S$, $\|x\|=1$, at least one of the line segments joining $x$ with $e$ and $-e$ is contained in the boundary of $S$.

Proof. Suppose $e=\frac{1}{2}(x+y), x, y \in S, x, y \neq e$. By the definition of semi- $L$-summand, there exists $x_{1}, y_{1} \in J^{\prime}, 0<\alpha, \beta<1$ such that

$$
x=\alpha x_{1}+(1-\alpha) e, \quad y=\beta y_{1}+(1-\beta) e .
$$

Hence $e=\left(\alpha x_{1}+\beta y_{1}\right) /(\alpha+\beta)$. This contradicts that $J$ is a semi- $L$ summand. The second part is clear by observing that the unit sphere of the subspace generated by $e$ and $x$ is a parallelogram.

THEOREM 5.4. Let $M$ be a semi-M-ideal in $X$. Then $M$ satisfies

$$
(1+\rho) S \cap \overline{(S+M)} \subseteq \overline{S+\rho S(M)}
$$

and hence it is a $U$-proximinal subspace in $X$.

Proof. Let $x \in(1+\rho) S \cap(S+M)$ with $\|x\|=1+\rho$. Note that $\langle M, x\rangle$ also has the 2-ball property. We may assume, without loss of generality, that $X=\langle M, x\rangle$. Then $J=M^{\perp}$ is a one dimensional semi- $L$-summand in $X^{*}$. Let $e^{*} \in J \cap S$. It follows from Lemma 5.3 and [13, p. 44, Theorem 4.7] that for any extreme point $x^{* *}$ in $S\left(X^{* *}\right), x^{* *}\left(e^{*}\right)=1$. Let

$$
F=\left\{x^{* *} \in S\left(X^{* *}\right): x^{* *}\left(e^{*}\right)=1\right\}
$$

$F \cup-F$ contains all extreme points of $S\left(X^{* *}\right)$. By the Krein-Milman theorem and the fact that $F$ is $w^{*}$-compact,

$$
\operatorname{conv}(F \cup-F)=\overline{\operatorname{conv}}^{w^{*}}(F \cup-F)=S\left(X^{* *}\right) \text {. }
$$

Hence Lemma 5.2 applies and

$$
\begin{aligned}
x \in\left(S\left(X^{* *}\right)+\rho\left(S\left(X^{* *}\right) \cap M^{* *}\right)\right) \cap X & =\overline{S(X)+\rho(S(X) \cap M)}^{w^{*}} \cap S \\
& \subseteq \overline{S(X)+\rho(S(X) \cap M)} .
\end{aligned}
$$

It follows that $(1+\rho) S \cap \overline{(S+M)} \subseteq \overline{S+\rho S(M)}$. Proposition 2.4 implies $M$ is a $U$-proximinal subspace with $\varepsilon(\rho)=2 \rho$.

6. Approximation by compact operators. Let $E, F$ be Banach spaces, we will use $L(E, F)(K(E, F))$ to denote the space of bounded linear operators (compact operators) from $E$ into $F$. In [4], [11], it is shown that if $E=l^{p}$, $F=i^{q}, 1<p, q<\infty$, then $K(E, F)$ is an $M$-ideal in $L(E, F)$. By Theorem 5.4, $K(E, F)$ is actually $U$-proximinal in $L(E, F)$. For the case $E=F=l^{1}$, 
Smith and Ward [16] and Mach and Ward [15] showed that $K\left(l^{1}, l^{1}\right)$ is not an $M$-ideal in $L\left(l^{1}, l^{1}\right)$; however, it is a proximinal subspace. In the following, we will consider a more general setting and that $K\left(l^{1}, l^{1}\right)$ is a $U$-proximinal subspace of $L\left(l^{1}, l^{1}\right)$ comes as a corollary.

LEMMA 6.1. Let $(W, \mathscr{B}, \sigma)$ be a positive measure space and suppose $f, g, h \in$ $L^{1}(W)$ satisfy $f=g+h,\|f\|<1+\rho,\|g\|<1$. Then there exist $g^{\prime}, h^{\prime} \in$ $L^{\prime}(W)$ such that $f=g^{\prime}+h^{\prime}$ with $\left\|g^{\prime}\right\|<1,\left\|h^{\prime}\right\|<8 \rho$ and $\left|h^{\prime}(x)\right|<|h(x)|$ for all $x \in W$.

Proof. We will assume that $1<\|f\|<1+\rho$ (otherwise, we can take $\left.g^{\prime}=f, h^{\prime}=0\right)$ and divide the measure space $W$ into three parts:

$$
\begin{aligned}
D_{1}= & \{x: g(x)>0, h(x)>0\} \cup\{x: g(x)<0, h(x)<0\}, \\
D_{2}= & \{x: g(x)>0, h(x)<0, f(x)<0\} \\
& \cup\{x: g(x)<0, h(x)>0, f(x)>0\},
\end{aligned}
$$

and

$$
\begin{aligned}
D_{3}= & \{x: g(x)>0, h(x)<0, f(x)>0\} \\
& \cup\{x: g(x)<0, h(x)>0, f(x)<0\} .
\end{aligned}
$$

Let $f / D$ denote the restriction of $f$ to $D$. It is clear that

$$
\begin{gathered}
\left\|f / D_{1}\right\|=\left\|g / D_{1}\right\|+\left\|h / D_{1}\right\|, \\
\left\|f / D_{2}\right\|=\left\|h / D_{2}\right\|-\left\|g / D_{2}\right\|,
\end{gathered}
$$

and

$$
\left\|f / D_{3}\right\|=\left\|g / D_{3}\right\|-\left\|h / D_{3}\right\|
$$

Three cases arise:

Case (i). If $\left\|h / D_{1}\right\| \geqslant \rho$, we let $h^{\prime}=\rho\left\|h \chi_{D_{1}}\right\|^{-1} h \chi_{D_{1}}$ and let $g^{\prime}=f-h^{\prime}$. Then it is easy to check that $\left\|g^{\prime}\right\| \leqslant 1,\left\|h^{\prime}\right\|=\rho$ and $\left|h^{\prime}(x)\right|<|h(x)|$ for all $x \in W$.

Case (ii). If $\left\|h / D_{1}\right\|<\rho$ and $\left\|h / D_{2}\right\|<3 \rho$, then we have

$$
\left\|f / D_{1}\right\|<\rho+\left\|g / D_{1}\right\|,\left\|f / D_{2}\right\|<3 \rho
$$

and

$$
\left\|f / D_{3}\right\|=\left\|g / D_{3}\right\|-\left\|h / D_{3}\right\| \text {. }
$$

Thus $1<\|f\| \leqslant 1+4 \rho-\left\|h / D_{3}\right\|$, which implies $\left\|h / D_{3}\right\|<4 \rho$ and hence $\|h\|<8 \rho$. For this case, we let $g^{\prime}=g, h^{\prime}=h$.

Case (iii). If $\left\|h / D_{1}\right\|<\rho$ and $\left\|h / D_{2}\right\| \geqslant 3 \rho$, then $\left\|f / D_{2}\right\|>\rho$. For otherwise, $\left\|f / D_{2}\right\|<\rho$ implies that $\left\|g / D_{2}\right\| \geqslant 2 \rho$. Thus $\left\|f / D_{2}\right\|<\rho<$ $\left\|g / D_{2}\right\|-\rho$ and it follows that

$$
\|f\| \leqslant\left(\left\|g / D_{1}\right\|+\rho\right)+\left(\left\|g / D_{2}\right\|-\rho\right)+\left\|g / D_{3}\right\|<1,
$$

which contradicts our assumption that $1<\|f\|$ and proves the claim. We 
define $h^{\prime}=\rho\left\|f \chi_{D_{2}}\right\|^{-1} f \chi_{D_{2}}$ and let $g^{\prime}=f-h^{\prime}$. Then for $x \in D_{2},\left|h^{\prime}(x)\right|<$ $|f(x)|<|h(x)|$ and hence $\left|h^{\prime}(x)\right|<|h(x)|$ for all $x \in W$. It is clear that $\left\|g^{\prime}\right\|=\|f\|-\rho<1$.

Let $(\Omega, \Sigma, \mu)$ be a $\sigma$-finite measure space and let $Y$ be a Banach space. A function $f: \Omega \rightarrow Y$ is called a Bochner measurable function if there exists a sequence of functions $\left\{f_{n}\right\}$ of the form $\sum_{i=1}^{\infty} y_{i} \chi_{E_{i}}$, where $y_{i} \in Y,\left\{E_{i}\right\}$ is a measurable partition of $\Omega$ and $\left\{f_{n}\right\}$ converges to $F$ uniformly except on a zero set. Let $L^{\infty}(\Omega, Y)$ denote the space of bounded Bochner measurable functions from $\Omega$ into $Y$ with norm defined by the essential supremum norm. It is clear from the definition that the set of countable valued Bochner measurable functions is dense in $L^{\infty}(\Omega, Y)$.

Let $(\Omega, \Sigma, \mu)$ be a $\sigma$-finite measure space and let $(W, \mathscr{B}, \sigma)$ be a positive measure space. We use $L_{\mathrm{wc}}^{\infty}\left(\Omega, L^{1}(W)\right)$ to denote the subspace of $L^{\infty}\left(\Omega, L^{1}(W)\right)$ consists of those functions $f$ such that $f(\Omega \backslash N)$ is a weak precompact set for some zero set $N$ in $\Omega$.

LEMMA 6.2. Let $H \in L_{\mathrm{wc}}^{\infty}\left(\Omega, L^{1}(W)\right)$ and let $H^{\prime} \in L^{\infty}\left(\Omega, L^{1}(W)\right)$ such that for almost all $\omega \in \Omega,\left|H^{\prime}(\omega)\right| \leqslant|H(\omega)|$. Then $H^{\prime} \in L_{\mathrm{wc}}^{\infty}\left(\Omega, L^{1}(W)\right)$.

Proof. It is well known that for any subset $K$ in $L^{1}(W, \mathscr{B}, \sigma), K$ is weakly precompact if and only if it is bounded and for any decreasing sequence $\left\{E_{n}\right\} \subseteq \mathscr{B}$ such that $\cap E_{n}=\varnothing,\left\{\int_{E_{n}} f d \sigma\right\}$ converges uniformly to zero for all $f \in K[2$, p. 292, p. 430]. This is also equivalent to $\{|f|: f \in K\}$ is weakly precompact. If $H \in L_{\mathrm{wc}}^{\infty}\left(\Omega, L^{1}(W)\right)$, then there exists a zero set $N_{1}$ such that $H\left(\Omega \backslash N_{1}\right)$ is weakly precompact. By hypothesis, $\left|H^{\prime}(\omega)\right|<|H(\omega)|$ for $\omega \in \Omega$ $\backslash N_{2}$ where $N_{2}$ is a zero set, it follows from the above remark that $H^{\prime}\left(\Omega \backslash\left(N_{1}\right.\right.$ $\left.\cup N_{2}\right)$ ) is also weakly precompact; therefore $H^{\prime} \in L_{\mathrm{wc}}^{\infty}\left(\Omega, L^{l}(W)\right)$.

THEOREM 6.3. $L_{\mathrm{wc}}^{\infty}\left(\Omega, L^{1}(W)\right)$ is a $U$-proximinal subspace of $L^{\infty}\left(\Omega, L^{1}(W)\right)$.

Proof. Let $X=L^{\infty}\left(\Omega, L^{1}(W)\right)$ and let $M=L_{\mathrm{wc}}^{\infty}\left(\Omega, L^{1}(W)\right)$, in view of Proposition 2.4(i), it suffices to show that

$$
(1+\rho) S \cap\left(S_{D}+M_{D}\right) \subseteq S+8 \rho S(M)
$$

where $S_{D}$ and $M_{D}$ denote the dense subsets of functions with countable values in $S$ and $M$, respectively. Let $F \in(1+\rho) S \cap\left(S_{D}+M_{D}\right)$; we can write $F=G+H$ with $\|G\| \leqslant 1, H \in M$ and

$$
\begin{aligned}
& G(\omega)(y)=\sum_{n=1}^{\infty} g_{n}(y) \cdot \chi_{E_{n}}(\omega), \\
& H(\omega)(y)=\sum_{n=1}^{\infty} h_{n}(y) \cdot \chi_{E_{n}}(\omega),
\end{aligned}
$$

where $\omega \in \Omega, y \in W$ and $\left\{E_{n}\right\}$ is a measurable partition of $E$. By Lemma 5.1, for each $n$, there exist $g_{n}^{\prime}$ and $h_{n}^{\prime}$ such that $\left\|g_{n}^{\prime}\right\|<1,\left\|h_{n}^{\prime}\right\|<8 \rho,\left|h_{n}^{\prime}\right|<|h|$ and 
$g_{n}+h_{n}=g_{n}^{\prime}+h_{n}^{\prime}$. Define $G^{\prime}, H^{\prime}$ for $\omega \in \Omega, y \in W$ by

$$
\begin{aligned}
& G^{\prime}(\omega)(y)=\sum_{n=1}^{\infty} g_{n}^{\prime}(y) \cdot \chi_{E_{n}}(\omega), \\
& H^{\prime}(\omega)(y)=\sum_{n=1}^{\infty} h_{n}^{\prime}(y) \cdot \chi_{E_{n}}(\omega) .
\end{aligned}
$$

It follows that $\left\|G^{\prime}\right\| \leqslant 1,\left\|H^{\prime}\right\| \leqslant 8 \rho$ and $\left|H^{\prime}(\omega)\right|<|H(\omega)|, \omega \in \Omega$. Hence $H^{\prime} \in M\left(\right.$ Lemma 6.2) and $F=G^{\prime}+H^{\prime} \in S+8 \rho S(M)$.

Let $(\Omega, \Sigma, \mu)$ be a $\sigma$-finite measure space and let $X$ be a Banach space with separable dual. For any operator $T: L^{1}(\Omega) \rightarrow X^{*}$, there exists a bounded Bochner measurable function $F: \Omega \rightarrow X^{*}$ such that

$$
\langle T(f), x\rangle=\int_{\Omega} f(\omega)\langle F(\omega), x\rangle d \mu(\omega), \quad x \in X,
$$

and $\|T\|=\|F\|_{\infty}$. Conversely, for any given bounded Bochner measurable function, we can define an integral operator $T$ with $F$ as kernel $[2$, p. 506]. We will identify $L\left(L^{1}(\Omega), X^{*}\right)$ and $L^{\infty}\left(\Omega, X^{*}\right)$. Moreover, it is known that $T$ is weakly compact if and only if the corresponding $F$ is in $L_{\mathrm{wc}}^{\infty}\left(\Omega, X^{*}\right)$.

THEOREM 6.4. Let $(\Omega, \Sigma, \mu)$ be a $\sigma$-finite measure space. Then $K\left(L^{1}(\Omega), l^{1}\right)$ is a U-proximinal subspace of $L\left(L^{1}(\Omega), l^{1}\right)$.

Proof. As is well known, weak sequential convergence and norm convergence are equivalent in $l^{1}$. Hence the Eberlein-Smulian theorem [2, p. 430] implies that weak compactness and norm compactness in $l^{1}$ are identical. Upon identifying $K\left(L^{1}(\Omega), l^{1}\right)$ with $L_{\mathrm{wc}}^{\infty}\left(\Omega, l^{1}\right)$, the result follows from Proposition 5.3.

COROllaRy 6.5. $K\left(l^{1}, l^{1}\right)$ is a $U$-proximinal subspace of $L\left(l^{1}, l^{1}\right)$.

To conclude this section, we remark that little is known about the proximinality of the subspace of compact operators on $L^{p}, 1<p<\infty$, $p \neq 2$ or $C(K)$. In [10] it was proved that if (i) $E=L^{1}(\mu)$ where $\mu$ is a $\sigma$-finite measure and $F$ is uniformly convex or (ii) $E^{*}$ is uniformly convex and $F=C(K)$ for some topological space $X$, then $K(E, F)$ is a proximinal subspace in $L(E, F)$. For operators between $l^{p}$ and $l^{q}, 1<p, q<\infty$, the only remaining unanswered case is $K\left(l^{\infty}, l^{p}\right), 1<p<\infty$.

\section{REFERENCES}

1. E. Alfsen and E. Effros, Structure in real Banach spaces, Ann. of Math: (2) 96 (1972), 98-173.

2. N. Dunford and J. Schwartz, Linear operators. I, Interscience Publishers, New York, 1958.

3. H. Fakhoury, Sur les $M$-idéaux dans certains espaces d'opérateurs et l'approximation par des opérateurs compacts (to appear).

4. J. Hennefeld, $A$ decomposition of $B(X)^{*}$ and unique Hahn-Banach extensions, Pacific J. Math. 46 (1973), 197-199. 
5. R. Holmes, M-ideals in approximation theory, Approximation Theory. II, Academic Press, New York, 1976, pp. 391-396.

6. R. Holmes and B. Kripke, Approximation of bounded functions by contimuous functions, Bull. Amer. Math. Soc. 71 (1965), 896-897.

7. __ Smoothness of approximation, Michigan Math. J. 15 (1968), 225-248.

8. __ Best approximation by compact operators, Indiana Univ. Math. J. 21 (1971), 255-263.

9. R. Holmes, B. Scranton and J. Ward, Approximation from the space of compact operators and other M-ideals, Duke Math. J. 42 (1975), $259-269$.

10. K. Lau, Approximation by contimuous vector valued functions, Studia Math. (to appear).

11. , M-ideals and approximation by compact operators (unpublished).

12. A. Lima, Intersection of balls and subspaces in Banach spaces, Trans. Amer. Math. Soc. 227 (1977), 1-62.

13. J. Lindenstrauss, Extension of compact operators, Mem. Amer. Math. Soc. No. 48, 1964.

14. __ On nonlinear projections in Banach spaces, Michigan Math. J. 11 (1964), 263-287.

15. J. Mach and J. Ward, Approximation by compact operators on certain Banach spaces, J. Approximation Theory (to appear).

16. R. Smith and J. Ward, M-ideal structure in Banach algebra, J. Functional Analysis 27 (1978), 337-349.

Department of Mathematics, University of Pittsburgh, Pittsburgh, Pennsylvania 15260 